\title{
Impact of Smoking on Circadian Typology, Sleep Habits and Mental Health of Japanese Students Aged 18 - 30 Years
}

\author{
Takahiro Kawada', Kotaro Oki², Yumiko Yamazaki², Fujiko Tsuji ${ }^{2,3}$, Miyo Nakade ${ }^{4}$, \\ Teruki Noji ${ }^{1}$, Milada Krejci ${ }^{3}$, Hitomi Takeuchi ${ }^{2}$, Tetsuo Harada ${ }^{2 *}$ \\ ${ }^{1}$ Center for Regional Collaboration, Kochi University, Kochi, Japan \\ ${ }^{2}$ Laboratory of Environmental Physiology, Graduate School of Integrated Arts and Sciences, Kochi University, \\ Kochi, Japan \\ ${ }^{3}$ University of Physical Education and Sports PALESTRA, Prague, Czech Republic \\ ${ }^{4}$ Faculty of Health and Nutrition, Tokai-Gakuen University, Nagoya, Japan \\ Email: "haratets@kochi-u.ac.jp
}

Received 15 March 2016; accepted 6 August 2016; published 9 August 2016

Copyright (C) 2016 by authors and Scientific Research Publishing Inc.

This work is licensed under the Creative Commons Attribution International License (CC BY).

http://creativecommons.org/licenses/by/4.0/

\section{(c) (i) Open Access}

\begin{abstract}
Many studies have shown that smoking can be related to worse sleep quality. However, as the impact of smoking, double view points on mental health and chronotype have been shortened. An integrated questionnaire including the diurnal type scale constructed by Torsvall \& Åkerstedt, and questions on smoking was administered to 1376 students attending university and mental training schools (693 women and 683 men), 19.9 years on average in 2010-2013. Only 4.1\% of the participants were smokers and they tended to be more evening-typed than non-smokers $(p=0.061)$. Smokers had significantly more difficulty falling asleep $(p=0.003)$ and more frequently lacked emotional control $(p=0.001)$ than non smokers. Smoking, being evening-type and having poor mental health may all be correlated in students attending Japanese university and medical training schools.
\end{abstract}

Keywords

Smoking, Circadian Typology, Sleep Quality, Late-Night TV

\section{Introduction}

Jaehne et al. (2009) described in a review that nicotine consumption influences mood and sleep by acting on "Corresponding author.

How to cite this paper: Kawada, T., Oki, K., Yamazaki, Y., Tsuji, F., Nakade, M., Noji, T., Krejci, M., Takeuchi, H., \& Harada, T. (2016). Impact of Smoking on Circadian Typology, Sleep Habits and Mental Health of Japanese Students Aged 18 - 30 Years. Psychology, 7, 1211-1216. http://dx.doi.org/10.4236/psych.2016.79122 
various neurotransmitter systems in synapses, and promotes lingered sleep latency, sleep fragmentation, decreased slow wave sleep, daytime sleepiness and suppression of rapid eye movement (REM) sleep. Sabanayagam and Shankar (2011) examined the association between smoking, smokeless tobacco use, second-hand smoke exposure and insufficient sleep in more than 100,000 individuals using the U.S. state-based 2008 Behavioral Risk Factor Surveillance System. Participants who were both current smokers and current smokeless cigarette users had twice the odds of insufficient sleep. Moreover, participants who were exposed to second-hand smoke showed a significant risk of insufficient rest/sleep.

Liu et al. (2013) investigated the impact of cigarette smoking on memory and sleep quality in 68 healthy participants using the Wechster Memory Scale Revised (Hunsley \& Lee, 2010) and a Chinese Pittsburgh Sleep Quality Index (Ho \& Fong, 2014). Smokers had poorer visual memory and poorer sleep quality than nonsmokers. Polisomnography (PSG) analysis was performed by Jaehne et al. (2012) and PSG sleep laboratory data was obtained from 44 smokers aged 29.6 years on average and compared to 44 non-smokers. Smokers showed a shorter sleep period, longer sleep latency, higher REM sleep density and more sleep apneas and leg movements in sleep than non-smokers. Moreover, plasma nicotine levels in smokers correlated negatively with amount of slow wave sleep. Brook et al. (2012) examined whether trajectories of cigarette smoking in adulthood predict insomnia among women in late mid-life using multivariate logistic regression analysis. Structured interviews at four time waves in adulthood (mean ages $=40$, 43, 48 and 65 years) showed that members of the chronic heavy smoking trajectory group were more likely to report insomnia at mean age 65 .

Hamidovic and Wit (2009) tested whether sleep deprivation promotes smoking behavior in healthy smokers using a two session within subject study after overnight sleep deprivation or after a normal night of sleep. Sleep deprivation increased fatigue, decreased arousal and increased the number of subjects who chose "cigarettes" instead of "money" as rewards to reduce the sleepiness.

Several studies have showed that smoking is related to poor sleep quality. Wittmann et al. (2006) examined the relationship between chronotype and smoking on 501 German volunteers and reported a striking correlation between chronotype and smoking, and smoking frequency is significantly higher in late chronotypes of all ages. The same research team analysed the same data base as Wittmann et al. (2006) used and concluded that the mediation analysis suggested that only those late chronotypes who smoke and those who drink more suffer from increased psychological distress (Wittmann et al., 2010). Randler (2008) also reported that smokers are more evening-typed and had a greater difference in wake-up times between week days and weekends than non-smokers. Nakade et al. (2009) reported also that smokers were more evening-typed and with lower sleep quality than non smokers in Japanese university students.

As the impact of smoking, double view points on mental health and chronotype have been shortened. It has been remained to be studied whether the worse mental health induced by smoking is direct effect of nicotine on the brain or indirect effect through the evening-typed life style which was induced by the smoking. This is the primary research question of this study.

On the other hand, all the epidemiological studies have been based on relatively small size of samples less than 700 and there have been no studies on large samplings more than 1000 on the relationship between chronotype and smoking. This study, secondly, aims to examine the relationship between smoking and circadian typology \& sleep habits of large sampling more than 1000 Japanese university students.

\section{Methods}

\subsection{Questionnaire Work and Participants}

An integrated questionnaire (Harada et al., 2012; Harada et al., 2013; Akimitsu et al., 2013) included a diurnal type scale (Torsvall \& Åkestedt, 1980; Harada et al., 2011) questions on sleep habits and sleep quality, questions on mental health (frequency of lacking emotional control, irritation, anger from a small trigger and depression), question on frequency of watching late-night TV and questions on smoking (Table 1). The questionnaire was administered to 1376 students in university or medical training school for physical therapists and medical nurses (693 women and 683 men) aged 18 to 30 years (more than 95\% 18 - 22 years) in May to October, 2010-2013. The response rates for this sample were $98 \%$ to $100 \%$.

\subsection{Statistic Analysis}

The questionnaire data was analyzed with SPSS 12.0 statistical software. Comparison between smokers and 
non-smokers was performed with Mann-Whitney U tests for continuous values. For categorical data, $\chi^{2}$-tests were used to analyze differences between smokers and non-smokers.

\subsection{Ethic Treatment}

The study followed the guidelines established by the Chronobiology International Journal for the conduct of research on human subjects (Portaluppi et al., 2010). Before administrating the questionnaires, each participant was given a written explanation that detailed the concepts and purposes of the study and stated that their answers would be used only for academic purposes. After the above explanation, all participants agreed completely with the proposal. The study was also permitted by the ethic committee in the Laboratory of Environmental Physiology, Graduate School of Integrated Arts and Sciences, Kochi University which carried out an ethical inspection of the contents of the questionnaire.

\section{Results}

Only $4.7 \%$ (females: $2.0 \%$, males: $7.5 \%$ ) of students attending university or medical training schools were smokers. Smokers were more evening-typed than non-smokers (Mann-Whitney U-test: $z=-2.402, p=0.016$ ) (Figure 1, Figure 2). Bedtime and wake-up times in both weekdays and weekend of smokers were significantly later than those of non-smokers (Table 2). The sleep duration both in weekdays and holidays was slightly longer than that of non-smokers (Table 2). Smokers had difficulty falling asleep both before weekdays and weekend more frequently than non-smokers both of females and males (Table 3(a), Table 3(c)). Moreover, smokers had difficulty awakening in weekdays more frequently than non-smokers (Table 3(b)). Frequency of smokers to feel tired in school was significantly higher than that of non-smokers (Table 3(d)). Smokers experienced a lack of

\section{Table 1. Multiple-choice question on smoking habits.}

\begin{tabular}{lllc}
\hline Q: & \multicolumn{1}{c}{ Do you smoke cigarettes? } \\
\hline A: & $(1)$ & Yes & $([1]<5$ cigarettes per day, [2] $6-10,[3] 11-20,[4] 21-30,[5] 31-40,[6]>40)$ \\
& $(2)$ & No & \\
\hline
\end{tabular}

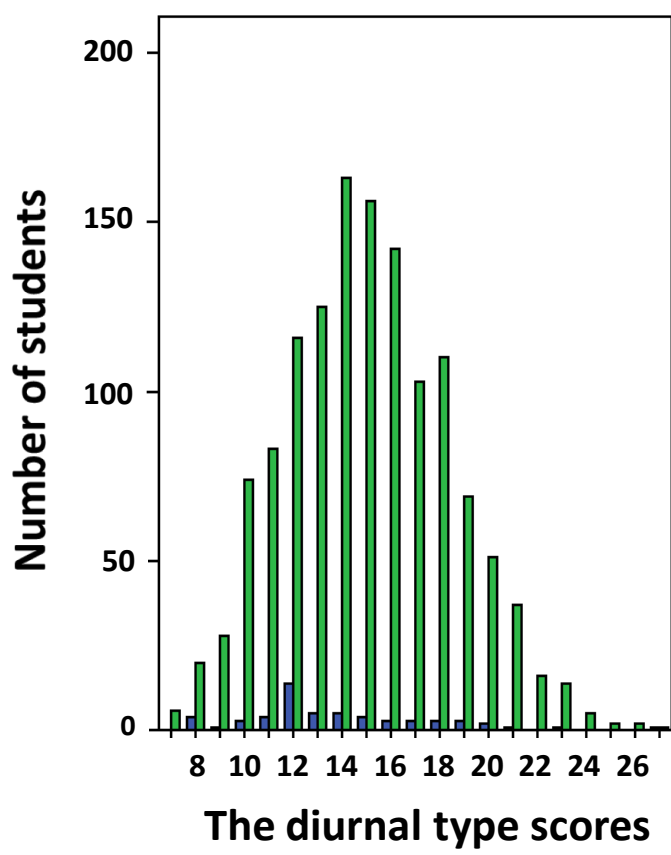

\section{: Yes \\ : No \\ Do you take \\ cigarettes?}

Figure 1. Comparison of the distribution of the diurnal type scores by smokers to that of non-smokers in Japanese students aged 18 - 30 years. 


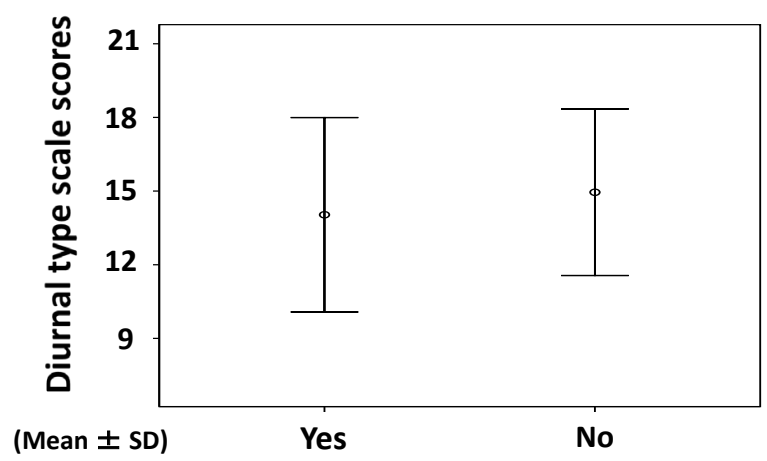

Do you take cigarettes?

(Mann-Whitney U-test: $\mathrm{z}=-\mathbf{2} .402, \mathrm{p}=0.016$ )

Figure 2. Smokers were significantly evening-typed than non-smokers of Japanese students aged 18 - 30 yrs.

Table 2. Comparison of sleep habits between smokers and non-smokers (Mean \pm SD).

\begin{tabular}{ccccccc}
\hline & \multicolumn{2}{c}{ Smokers } & \multicolumn{3}{c}{ Non-smokers } & \multicolumn{2}{c}{ Mann-Whitney U-test } \\
\hline & & & & z & P \\
WD Bedtime & $24.9 \pm 1.2$ & $(68)$ & $24.5 \pm 1.2$ & $(1286)$ & -2.304 & 0.021 \\
WE Bedtime & $25.9 \pm 1.5$ & $(67)$ & $25.2 \pm 1.5$ & $(1312)$ & -3.749 & $<0.001$ \\
WD Wake up time & $7.8 \pm 1.0$ & $(70)$ & $7.3 \pm 0.9$ & $(1406)$ & -4.880 & $<0.001$ \\
WE Wake up time & $10.4 \pm 2.2$ & $(69)$ & $9.4 \pm 1.7$ & $(1411)$ & -1.478 & $<0.001$ \\
WD Sleep hours & $6.9 \pm 1.2$ & $(68)$ & $6.7 \pm 1.2$ & $(1278)$ & -1.975 & 0.048 \\
WE Sleep hours & $8.6 \pm 1.7$ & $(67)$ & $8.2 \pm 1.6$ & $(1311)$ & -2.511 & 0.012
\end{tabular}

Table 3. Sleep quality and tiredness in daytime of smokers and non-smokers.

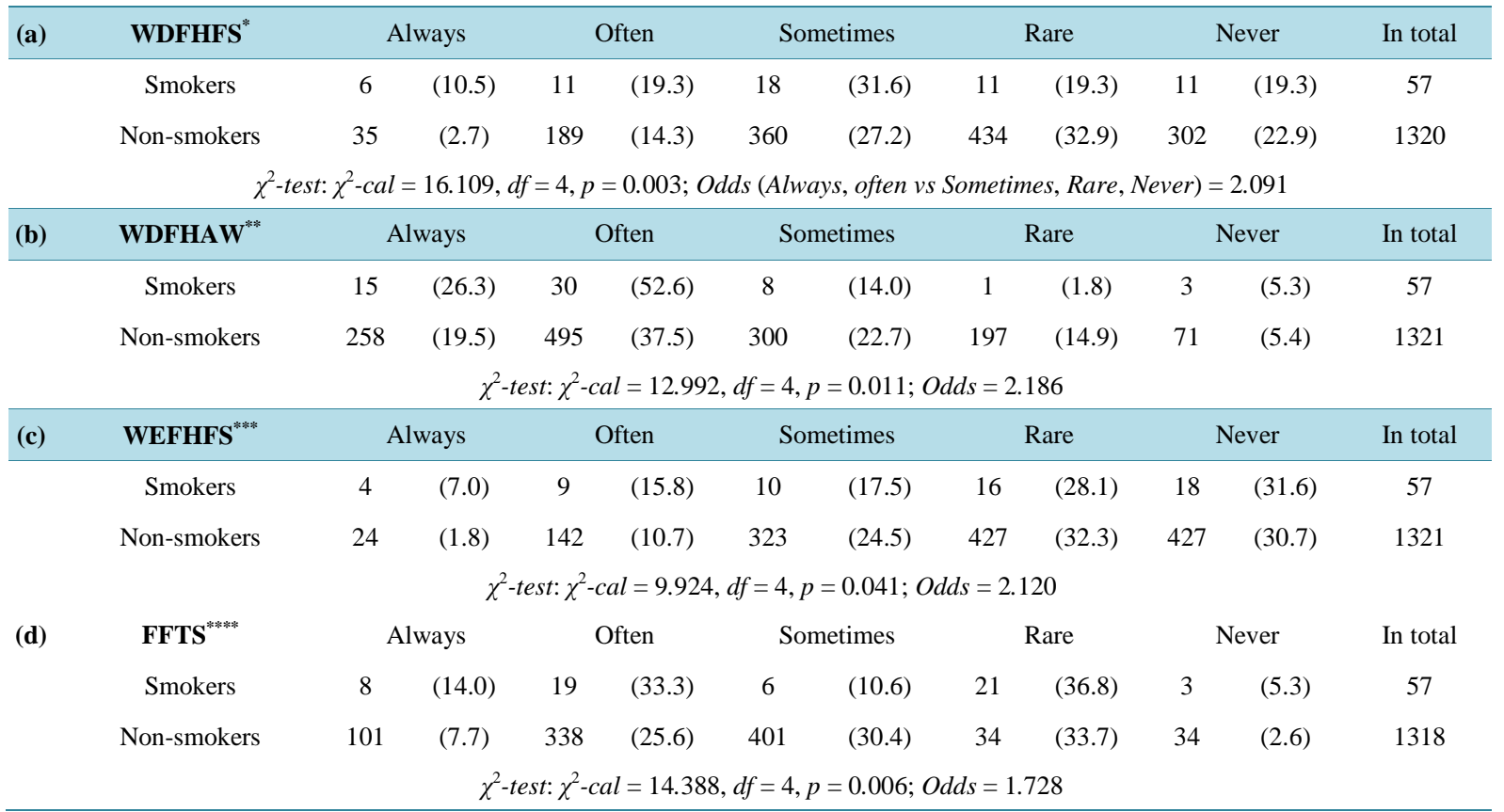

*WDFHFS: Week day frequency to have difficulty for falling sleep; ${ }^{* *}$ WDFHAW: Week day frequency to have difficulty for awakening; ${ }^{* * *}$ WEFHFS: Week end frequency to have difficulty for falling sleep; ${ }^{* * *}$ FFTS: Frequency to feel tired at school. 


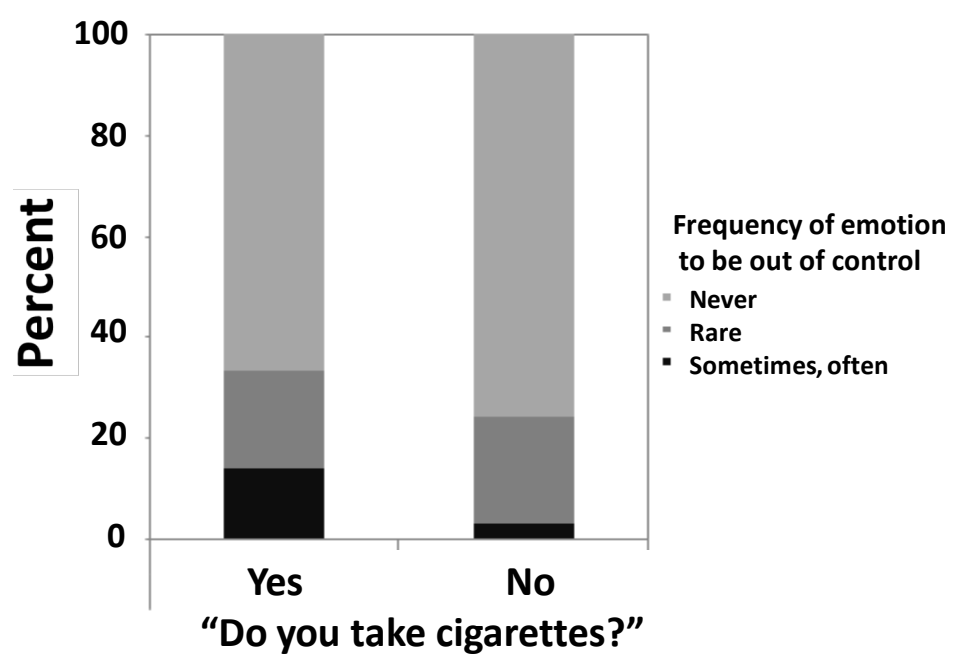

Figure 3. Smokers lack emotional control more frequently than nonsmokers in Japanese students aged 18 - 30 years.

emotional control more frequently than non-smokers $\left(\chi^{2}\right.$-test: $\chi^{2}$-cal $\left.=16.679, d f=2, p<0.001\right)$ (Figure 3). Smokers had a lower mental health index score (total evaluation of four characteristics: lack of emotional control, irritation, anger and depression) than non-smokers (11.36 \pm 2.88 [70] for smokers vs. $12.01 \pm 2.30$ [1409] for non-smokers; one-way ANOVA: $F=5.164, \mathrm{df}=1, p=0.023)$.

In smokers and non-smokers altogether, evening-typed students (with diurnal type scores ranging from 7 to 12 points, mean \pm SD [n]: $11.58 \pm 2.35$ [367]) had lower mental health index scores than medium-typed students (13 to 17 points, $12.07 \pm 2.18$ [766]) and morning-typed students (18 to 28 points, $12.19 \pm 2.56$ [350]; KruskalWallis test: $\chi^{2}$-value $=21.30, d f=2, p<0.001$ ). The diurnal type scores were weakly, significantly and positively correlated with the mental health scores in the total data (morning types had better mental health overall; Pearson's correlation analysis: $r=0.099, n=1486, p<0.001$ ).

\section{Discussion}

In Japanese students aged 18 - 30 years, smoking appears to be related to being evening-typed, lower quality of sleep, shorter sleep duration and poorer mental health. So far, several studies have shown that healthy smokers have a poorer quality of sleep and shorter sleep duration. For example, a recent population-based case-control study in Germany on 1071 healthy smokers and 1243 healthy non-smokers showed an elevated prevalence of sleep disturbance in smokers compared with non-smokers (Cohrs et al., 2014). As another example, Lien et al. (2009) showed that there was a statistically significant association between daily smoking at age 15 and mental distress at age 18 for girls, but not for boys (odds ratio 2.0 [1.5 - 2.8] and 1.3 [0.7 - 2.4], respectively). In girls, the association remained statistically significant even after adjustment for sociodemographic variables and several lifestyle factors in the adolescents of Norway. To the best of our knowledge, however, this study is the first to report on the relationship between the smoking habits and an evening-typed lifestyle for the large samples more than 1000. ANCOVA analysis showed a significant relationship between smoking and poor mental health even with diurnal type as a covariant (relationship between smoking/non-smoking and mental health index score with diurnal type (morning-type, medium type and evening type) as a covariant: $F=3.899, d f=1, p=0.049$ ). This would indicate that the smoking habit by students with poorer mental health may not be via the evening-typed lifestyle which can be possible to induce worse mental health of smokers, but direct effect on worse mental health.

\section{Acknowledgements}

We thank all the participants of this study. Thanks are also due for the financial support from the Research Foundation by Japan Dairy Association (J-MILK) (2013-2014) (To T. HARADA). This MS has been checked by Ms Laura Sato, a professional English editor, from linguistic point of view. 


\section{References}

Akimitsu, O., Wada, K., Noji, T., Taniwaki, N., Krejci, M, Takeuchi, H., \& Harada, T. (2013). The Relationship between Consumption of Tyrosine and Phenylalanine as Precursors of Catecholamine at Breakfast and the Circadian Typology and Mental Health in Japanese Infants Aged 2 to 5 Years. Journal of Physiological Anthropology, 32, MS No. 13 1-8. http://dx.doi.org/10.1186/1880-6805-32-13

Brook, D. W., Bubenstone, E., Zhang, C., \& Brook, J. S. (2012). Trajectories of Cigarette Smoking in Adulthood Predict Insomnia among Women in Late Mid-Life. Sleep Medicine, 13, 1130-1137. http://dx.doi.org/10.1016/j.sleep.2012.05.008

Cohrs, S., Rodenbeck, A., Riemann, D., Szagun, B., Jaehne, A., Brinkmeyer, J., Gründer, G., Wienker, T., Diaz-Lacava, A., Mobascher, A., Dahmen, N., Thuerauf, N., Kornhuber, J., Kiefer, F., Gallinat, J., Wagner, M., Kunz, D., Grittner, U., \& Winterer, G. (2014). Impaired Sleep Quality and Sleep Duration in Smokers-Results from the German Multicenter Study on Nicotine Dependence. Addiction Biology, 19, 486-496. http://dx.doi.org/10.1111/j.1369-1600.2012.00487.x

Hamidovic, A., \& de Wit, H. (2009). Sleep Deprivation Increases Cigarette Smoking. Pharmacology Biochemistry and Behavior, 93, 263-269. http://dx.doi.org/10.1016/j.pbb.2008.12.005

Harada, T., Kobayashi, R., Wada, K., Nishihara, R., Kondo, A., Akimitsu, O., Noji, T., Taniwaki, N., Nakade, M., Krejci, M., \& Takeuchi, H. (2011). Effect of Birth Season on Circadian Typology Appearing in Japanese Young Children Aged 2 to 12 Years Disappears in Older Students Aged 18 to 25 Years. Chronobiology International, 28, 638-642. http://dx.doi.org/10.3109/07420528.2011.597529

Harada, T., Nakade, M., Wada, K., Akimitsu, O., Noji, T., Krejci, M., \& Takeuchi, H. (2013). Chapter 33. Tryptophan and Sleep: Breakfast Tryptophan Content and Sleep. In V. R. Preedy, V. B. Patel, \& L.-A. Le (Eds.), Handbook of Nutrition, Diet and Sleep (pp. 473-487, 500). Wageningen: Wageningen Academic Publishers.

Harada, T., Nakade, M., Wada, K., Kondo, A., Maeda, M., Noji, T., \& Takeuchi, H. (2012). Chapter 22. Mental Health of Children from a Chronobiological and Epidemiological Point of View. In V. Olisah (Ed.), Essential Notes in Psychiatry (pp. 439-458, 580). Rijeka: In Tech.

Ho, R. T., \& Fong, T. C. (2014). Factor Structure of the Chinese Version of the Pittsburgh Sleep Quality Index in Breast Cancer Patients. Sleep Medicine, 15, 565-569. http://dx.doi.org/10.1016/j.sleep.2013.10.019

Hunsley, J., \& Lee, C. M. (2010). Introduction to Clinical Psychology: An Evidence-Based Approach. New York: John Wiley \& Sons, Inc.

Jaehne, A., Loessl, B., Bárkai, Z., Riemann, D., \& Hornyak, M. (2009). Effects of Nicotine on Sleep during Consumption, Withdrawal and Replacement Therapy. Sleep Medicine Reviews, 13, 363-77. http://dx.doi.org/10.1016/j.smrv.2008.12.003

Jaehne, A., Unbehaun, T., Feige, B., Lutz, U. C., Batra, A., \& Riemann, D. (2012). How Smoking Affects Sleep: A Polysomnographical Analysis. Sleep Medicine, 13, 1286-1292. http://dx.doi.org/10.1016/j.sleep.2012.06.026

Lien, L., Sagatun, Å., Heyerdahl, S., Søgaard, A. J., \& Bjertness, E. (2009). Is the Relationship between Smoking and Mental Health Influenced by Other Unhealthy Lifestyle Factors? Results from a 3-Year Follow-Up Study among Adolescents in Oslo, Norway. Journal of Adolescent Health, 45, 609-617. http://dx.doi.org/10.1016/j.jadohealth.2009.04.011

Liu, J.-T., Lee, I.-H., Wang, C.-H., Chen, K.-C., Lee, C.-I., \& Yang, Y.-K. (2013). Cigarette Smoking Might Impair Memory and Sleep Quality. Journal of the Formosan Medical Association, 112, 287-290.

http://dx.doi.org/10.1016/j.jfma.2011.12.006

Nakade, M., Takeuchi, H., Kurotani, M., \& Harada, T. (2009). Effects of Meal Habits and Alcohol/Cigarette Consumption on Morningness-Eveningness Preference and Sleep Habits by Japanese Female Students Aged 18-29. Journal of Physiological Anthropology, 28, 83-90. http://dx.doi.org/10.2114/jpa2.28.83

Portaluppi, F., Smolensky, M. H., \& Touitou, Y. (2010). Effects and Methods for Biological Rhythm Research on Animals and Human Beings. Chronobiology International, 27, 1911-1929. http://dx.doi.org/10.3109/07420528.2010.516381

Randler, C. (2008). Differences between Smokers and Non-Smokers in Morningness-Eveningness. Social Behavior and Personality, 36, 673-680. http://dx.doi.org/10.2224/sbp.2008.36.5.673

Sabanayagam, C., \& Shankar, A. (2011).The Association between Active Smoking, Smokeless Tobacco, Second-Hand Smoke Exposure and Insufficient Sleep. Sleep Medicine, 12, 7-11. http://dx.doi.org/10.1016/j.sleep.2010.09.002

Torsvall, L., \& Åkerstedt, T. A. (1980). Diurnal Type Scale: Construction, Consistency and Validation in Shift Work. Scandinavian. Journal of Work, Environment and Health, 6, 283-290. http://dx.doi.org/10.5271/sjweh.2608

Wittmann, M., Dinich, J., Merrow, M., \& Roenneberg, T. (2006). Social Jetlag: Misalignment of Biological and Social Time. Chronobiology International, 23, 497-509. http://dx.doi.org/10.1080/07420520500545979

Wittmann, M., Paulus, M., \& Roenneberg, T. (2010). Decreased Psychological Well-Being in Late “Chronotypes” Is Mediated by Smoking and Alcohol Consumption. Substance Use \& Misuse, 45, $15-30$.

http://dx.doi.org/10.3109/10826080903498952 


\section{Submit or recommend next manuscript to SCIRP and we will provide best service for you:}

Accepting pre-submission inquiries through Email, Facebook, LinkedIn, Twitter, etc.

A wide selection of journals (inclusive of 9 subjects, more than 200 journals)

Providing 24-hour high-quality service

User-friendly online submission system

Fair and swift peer-review system

Efficient typesetting and proofreading procedure

Display of the result of downloads and visits, as well as the number of cited articles

Maximum dissemination of your research work

Submit your manuscript at: http://papersubmission.scirp.org/ 